\section{Análise das barreiras à utilização de trombolíticos em casos de acidente vascular cerebral isquêmico em um hospital privado do Rio de Janeiro, Brasil}

\author{
Analysis of barriers to the use of thrombolytics \\ in ischemic stroke in a private hospital in \\ Rio de Janeiro, Brazil
}

Luiz Henrique de Oliveira Fonseca ${ }^{1}$ Maria Luiza Garcia Rosa 2 Arthur Carvalho Silva 2 Renan Marchesi Maciel 2 André Volschan 1 Evandro Tinoco Mesquita ${ }^{3}$

\section{Resumo}

\footnotetext{
1 Hospital Pró-Cardíaco, Rio de Janeiro, Brasil.

2 Departamento de

Epidemiologia e Estatística,

Universidade Federal

Fluminense, Niterói, Brasil.

3 Departamento de Medicina

Clínica, Universidade Federal

Fluminense, Niterói, Brasil.

Correspondência

M. L. G. Rosa

Departamento de

Epidemiologia e Estatística,

Universidade Federal

Fluminense.

Rua Marques do Paraná,

303 anexo, 3 o andar, sala 6 ,

Niterói, RJ 24033-900, Brasil.

mluizagr@gmail.com
}

\begin{abstract}
Ischemic stroke is a major cause of neurological sequelae and death. The correct use of thrombolytic therapy faces several barriers. The objective was to discuss barriers to thrombolytic therapy in a private hospital. This was a retrospective cohort study of patients with symptoms of acute ischemic stroke entering the emergency department of a private hospital in Rio de Janeiro, Brazil, from 2009 to 2011. 257 patients were admitted with suspected ischemic stroke. Among the 156 patients with confirmed diagnosis of ischemic stroke, 18 (11.5\%) received thrombolytic therapy. Of the 30 patients with ischemic stroke within the therapeutic window and meeting NIHSS criteria for thrombolysis, 20 were not thrombolysed. Nine of these were due to administrative barriers (45\%). In the current study, the thrombolysis rate was higher than in the United States, but barriers prevented treating $47.6 \%$ of patients with indication for thrombolysis. Importantly, this study was conducted in a private hospital, and the situation may be even more unfavorable in the public health system.
\end{abstract}

Stroke; Thrombolytic Therapy; Quality of Health Care
O acidente vascular cerebral isquêmico (AVCi) é uma importante causa de sequela e morte. A correta utilização do trombolítico enfrenta várias barreiras. O objetivo foi discutir as barreiras à terapia trombolítica em pacientes que chegam, com sintomas de AVCi agudo, à emergência de um hospital privado do Rio de Janeiro, Brasil. Coorte retrospectiva de pacientes entre $2009 e$ 2011. Foram admitidos 257 pacientes com suspeita de AVCi. Dos pacientes com diagnóstico confirmado (156), 11,5\% (18) foram trombolisados. Dos 30 pacientes com diagnóstico de AVCi, dentro da janela terapêutica e com NIHSS na faixa para trombólise, 20 não foram trombolisados, 9 por barreiras administrativas (45\%). Neste trabalho o percentual de trombólise foi superior ao observado nos Estados Unidos, mas barreiras impediram o tratamento de 45\% dos pacientes com indicação à trombólise. Imagina-se que na rede pública brasileira a situação seja ainda mais desfavorável.

Acidente Vascular Cerebral; Terapia Trombolítica; Qualidade da Assistência à Saúde 


\section{Introdução}

O acidente vascular cerebral (AVC) é uma das principais causas de morte no mundo, sendo a principal no Brasil, e a principal causa de dependência funcional no Brasil e no mundo 1,2,3. Há dois tipos distintos de $\mathrm{AVC}$, hemorrágico ou isquêmico; o último com incidência muito superior ao primeiro 4 . Não só a prevenção representa um desafio à saúde pública, mas também o tratamento adequado, capaz de reduzir as incapacidades.

A correta indicação e utilização do trombolítico ativador do plasminogênio tissular recombinante (rtPA), cujo nome genérico é Alteplase, diminui a morbidade em $30 \%$. Em 2001, a Alteplase (rtPA) foi aprovada pelo Ministério da Saúde para o uso no AVC isquêmico (AVCi) no Brasil. Um dos primeiros consensos sobre o uso do trombolítico foi publicado em 2002 pela Sociedade Brasileira de Doenças Cerebrovasculares 5 e sua utilização é preconizada em diretrizes nacionais e internacionais 6,7. Em maio de 2010, a Alteplase foi incluída na lista de medicações essenciais do Sistema Único de Saúde (SUS) para o tratamento do AVC, e em abril de 2012 foram aprovados um protocolo clínico e diretrizes para trombólise no AVCi pelo Ministério da Saúde.

O tratamento com a rtPA é subutilizado no mundo todo 8,9,10, inclusive no Brasil 11,12 seja por extrapolar o tempo máximo para administração de 4,5 horas após o início dos sintomas (janela terapêutica 13), seja por ser desconhecida (liberação relativamente recente), ou por aumentar a probabilidade de hemorragia 7.

As diretrizes clínicas, uma vez corretamente implementadas, são consideradas importantes ferramentas para a melhoria da qualidade do cuidado ao AVCi, diminuindo a inadequada variação de prática e acelerando a incorporação de avanços na prática diária 14,15 . Uma extensa literatura, em vários países, tem tentado compreender quais os fatores que limitam a adesão dos médicos às diretrizes 16,17,18, apontando barreiras organizacionais, de fatores relacionados a pacientes, ao processo de implementação da diretriz e suas características.

Cabana et al. 16, com uma revisão sistemática da literatura publicada em 1999, propuseram uma classificação de barreiras que foi corroborada posteriormente por Cochrane et al. 17 , em outra revisão publicada em 2007. Identificaram sete categorias de barreiras ligadas diretamente aos médicos e três que chamaram de barreiras externas 16 .

O presente artigo visa à discussão das barreiras à terapia trombolítica em pacientes que chegam, com sintomas de AVCi agudo, à emergência de um hospital privado do Rio de Janeiro.

\section{Método}

Trata-se uma coorte retrospectiva de pacientes que deram entrada na emergência de um hospital privado da cidade do Rio de Janeiro, entre janeiro de 2009 e outubro de 2011. Os prontuários de todos pacientes com suspeita clínica de AVC (hipótese diagnóstica de AVC registrada em prontuário) foram revistos pelo autor principal do estudo, e os dados foram registrados em formulário previamente elaborado. As seguintes informações foram colhidas: idade, gênero, fatores de risco - diagnóstico médico anterior de hipertensão arterial sistêmica (HAS), diabetes mellitus, fibrilação atrial, AVC e doença arterial coronariana -, tempo de início de sintomas, pressão arterial, frequência cardíaca, glicemia capilar de admissão, tempo até a realização do primeiro exame de imagem - ressonância magnética ou tomografia computadorizada -, tempo de avaliação pelo neurologista, tipo de alta (alta, transferência e óbito) e indicação de trombólise, além de elementos do exame neurológico para escore NIHSS (National Institute of Health Stroke Scale). O NIHSS é uma escala que avalia quantitativamente as principais funções neurológicas de um paciente. Foi criada com o objetivo de avaliar os déficits neurológicos em pacientes na fase aguda de AVC, variando de 0 a 42 pontos, distribuídos em 11 categorias: nível de consciência, motricidade ocular, campos visuais, paresia facial, motor do membro superior, motor do membro inferior, ataxia apendicular, sensibilidade, linguagem, disartria e extinção/negligência.

Essas informações foram analisadas pelo pacote estatístico SPSS, versão 17.0 (SPSS Inc., Chicago, Estados Unidos). Trata-se de um estudo essencialmente descritivo e o número de pacientes incluídos (257) foi semelhante ao de outros estudos sobre o tema 19,20.

Os pacientes foram classificados em: (1) pacientes elegíveis para trombólise; (2) pacientes não elegíveis para trombólise por falta de indicação ou por presença de contraindicação clínica; (3) pacientes não elegíveis para trombólise por barreiras relacionadas diretamente aos médicos e barreiras externas.

Foram considerados pacientes elegíveis para trombólise aqueles com diagnóstico clínico de AVC, satisfazendo os critérios: (1) com NIHSS entre 4 e 21, sem melhora 7; (2) cujo exame de imagem foi compatível com AVCi (tomografia computadorizada normal e/ou ressonância magnética de crânio compatível com isquemia - pacientes, em princípio, com indicação à trombólise); (3) sem contraindicações clínicas 7; (4) com tempo total (tempo do início dos sintomas, tempo de primeira imagem e tempo de avaliação 
do neurologista) inferior a 3 ou 4 horas e meia, dependendo de quando foi admitido o paciente 13; (5) cujo médico assistente, familiar e paciente concordaram com o procedimento.

Foram classificadas como não elegíveis devido à não indicação de trombólise: (1) idade inferior a 18 anos; (2) tempo de início de sintomas superior à janela terapêutica inicialmente de 3 horas (até junho de 2009 e posteriormente 4,5 horas) ou paciente e/ou familiar não sabiam informar; (3) NIHSS menor que 4 e maior que 21; (4) melhora do NIHSS dentro da janela terapêutica; (5) não confirmação do diagnóstico de AVCi. As contraindicações clínicas foram as clássicas para o uso de trombolíticos no AVC 7 .

Foram identificadas como barreiras as ocorrências que impediram a trombólise. Foram classificadas como proposto por Cabana et al. 16. As barreiras ligadas diretamente aos médicos foram associadas, pelos autores, ao conhecimento e às atitudes: (1) falta de familiaridade; (2) falta de conhecimento; (3) falta de acordo com orientação específica; (4) falta de confiança nos autores da diretriz; (5) falta de expectativas de resultados; (6) falta de autoconfiança; (7) falta de motivação. As barreiras externas que influenciam as atitudes e o conhecimento, implicando comportamentos foram: (1) fatores relacionados aos pacientes; (2) fatores relacionados à diretriz e fatores ambientais (falta de tempo, falta de recursos, restrições organizacionais, falta de reembolso, aumento percebido de responsabilização por má prática).

A janela terapêutica - tempo do início dos sintomas até a trombólise - foi de 3 horas de janeiro a junho de 2009 e de 4,5 horas a partir de julho de 2009, conforme as recomendações do estudo de Hacke et al. 13.

Ressalta-se que toda a equipe do serviço de emergência do hospital estudado recebe treinamento periódico sobre os protocolos de atendimento, incluindo AVC.

Os resultados foram descritos em duas perspectivas: a primeira objetivando avaliar a capacidade do serviço de seguir o protocolo em cada uma das etapas, de forma prospectiva, partindo da chegada à emergência de todos os pacientes com suspeita clínica de AVC, indicando as exclusões por não indicação ou contraindicação clínica e por barreiras diretamente ligadas aos médicos e/ou barreiras externas (Figura 1); a segunda perspectiva, com resultados apresentados na Tabela 1, identifica as barreiras sem considerar se o paciente era ou não candidato à trombólise no momento em que a barreira foi identificada, podendo ocorrer mais de uma barreira para cada paciente.

O trabalho de pesquisa com uso dos prontuários foi avaliado pelo Comitê de Ética em Pesqui- sa do hospital estudado em 25 de novembro de 2009 (parecer no 332).

O hospital onde o presente estudo se desenvolveu, é um hospital terciário, focado na alta complexidade de assistência com cerca de 130 leitos. É uma instituição com acreditação hospitalar nacional e internacional. Possui uma emergência, serviço de atendimento pré-hospitalar e presta atendimento domiciliar, o que permite iniciar qualquer atendimento precocemente. A emergência é organizada para atender ao paciente com AVC de modo sistematizado e rápido com neurologistas de plantão 24 horas e equipe multidisciplinar. A equipe multidisciplinar da emergência é integralmente treinada para seguir o protocolo próprio do hospital que é baseado no guideline da American Stroke Association/ American Heart Association, e para realização do NIHSS. Todos os profissionais médicos são certificados ao fim do treinamento, revalidado regularmente. A população atendida tem bom nível educacional e de renda. Trata-se de um cenário muito favorável à aplicação da diretriz, em que espera-se que haja uma frequência baixa de barreiras. O paralelo com a situação do SUS permitirá a sugestão de iniciativas visando à superação de barreiras, também nessa rede.

\section{Resultados}

Um total de 257 pacientes foi admitido com suspeita clínica de AVC na emergência do hospital estudado entre janeiro de 2009 e outubro de 2011. A média do tempo entre a chegada do paciente e o primeiro atendimento foi de oito minutos. A frequência de homens foi ligeiramente superior a de mulheres (53,3\%), e a média de idade foi de 76,34 anos $( \pm 12,69)$. A maioria era hipertensa e não apresentou outras comorbidades. Destacase que cerca de $20 \%$ eram diabéticos e $22 \%$ referiram história prévia de fibrilação atrial. Houve cerca de $5 \%$ de óbitos hospitalares (Tabela 2) e somente um entre os 18 trombolisados morreu, nenhum dos 11 que foram trombolisados dentro dos critérios de elegibilidade evoluiu com óbito (Tabela 2).

A média (DP) de tempo até realização da primeira imagem foi de 79,64 $(108,86)$ e a mediana foi de 53 minutos. Em relação à avaliação do neurologista, a média (DP) foi de 237,20 $(371,53)$ e a mediana de 95 minutos. A correlação de Spearman entre o NIHSS e tempo da chegada até a primeira imagem foi de $-0,478$ (valor de $\mathrm{p}<0,001$ ) e entre NIHSS e tempo de avaliação do neurologista foi de $-0,326$ (valor de $\mathrm{p}<0,001$ ). A correlação da idade com tempo da chegada até a primeira imagem foi de 0,051 (valor de $p=0,423$ ) e com o 


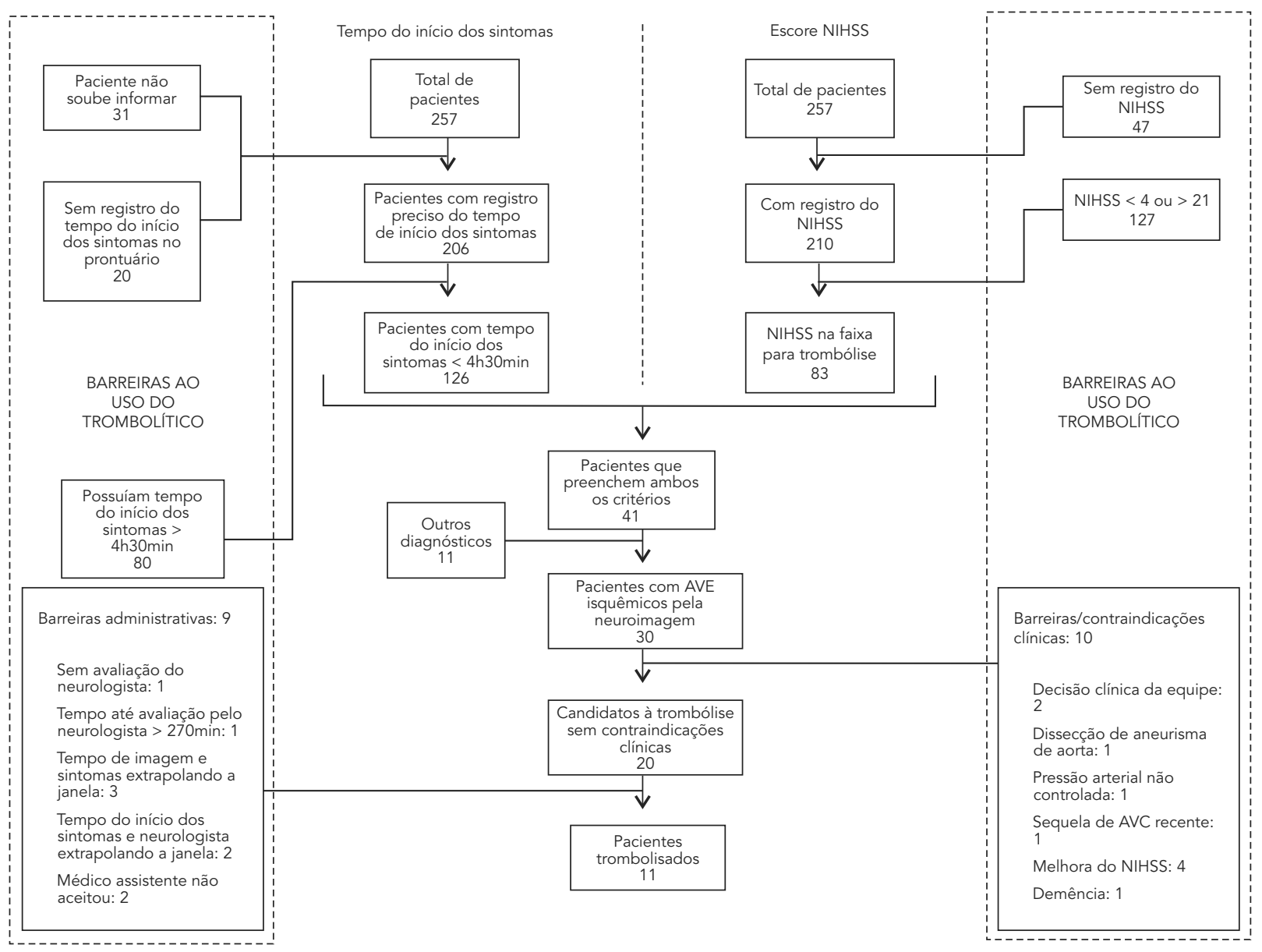

AVC: acidente vascular cerebral; NIHSS: National Institute of Health Stroke Scale.

tempo de avaliação do neurologista foi de $-0,010$ (valor de $\mathrm{p}=0,894$ ). Tais resultados indicam que quanto mais grave o paciente, menor o tempo de exame e que eles não estão correlacionados com a idade.

O fluxograma descreve o caminho do paciente da chegada ao hospital ao momento da administração do trombolítico, apontando as não indicações/contraindicações clínicas à trombólise, além das barreiras diretamente ligadas ao médico e as externas. Houve 11 pacientes sem indicação à trombólise, cuja neuroimagem não foi sugestiva de AVCi e 137 contraindicações clínicas (127 devido ao NIHSS ser maior que 21 ou menor que 4 e 10 por diferentes condições clínicas). Dos 20 pacientes com dados completos com indica- ção e sem contraindicação clínica à trombólise ( 14 homens e 6 mulheres), somente 11 (7 homens e 4 mulheres) receberam a medicação. Nove pacientes enfrentaram barreiras, dois casos por fatores ligados diretamente ao médico e sete casos por barreiras externas ligadas ao ambiente. Entre homens, o percentual de barreiras foi de $50 \%$ e entre mulheres de 33,3\% (valor de $\mathrm{p}=0,642$ ).

Do total de 18 pacientes trombolisados, 11 tinham indicação clínica e 7 foram trombolisados por decisão da equipe médica apesar da presença de não indicação/contraindicações clínicas NIHSS muito elevado (5), NIHSS de 22 (1) e paciente jovem com piora clínica (1).

O percentual de trombólise entre os 156 pacientes com o diagnóstico confirmado de AVCi 
Tabela 1

Barreiras ao trombolítico em pacientes atendidos em emergência com suspeita de acidente vascular cerebral isquêmico (AVCi). Rio de Janeiro,

Brasil ( $\mathrm{N}=257)$.

\begin{tabular}{|c|c|}
\hline Barreiras à trombólise & n (\%) \\
\hline \multicolumn{2}{|l|}{ Barreiras ligadas diretamente aos médicos } \\
\hline Prontuários não continham nenhuma referência ao tempo do início dos sintomas & $20(7,78)$ \\
\hline Médico assistente não permitiu a administração do trombolítico & $2(0,78)$ \\
\hline \multicolumn{2}{|l|}{ Barreiras externas } \\
\hline Prontuários indicando que paciente e/ou familiar não souberam informar momento do início dos sintomas & $31(12,06)$ \\
\hline Paciente sem registro ou com registro incompleto do NIHSS & $47(18,29)$ \\
\hline Tempo até avaliação do neurologista maior que 270 minutos & $51(19,84)$ \\
\hline Soma do tempo do início dos sintomas e primeira imagem maior que 270 minutos & $92(35,80)$ \\
\hline Soma do tempo da primeira imagem e neurologista maior que 270 minutos & $62(24,12)$ \\
\hline
\end{tabular}

NIHSS: National Institute of Health Stroke Scale.

Tabela 2

Características dos pacientes atendidos em emergência com suspeita de acidente vascular cerebral isquêmico (AVCi). Rio de Janeiro, Brasil (valores em frequência absoluta e relativa, ou como indicado).

\begin{tabular}{|c|c|c|c|c|}
\hline \multirow[t]{3}{*}{ Características } & \multicolumn{4}{|c|}{ Ano do atendimento } \\
\hline & 2009 & 2010 & 2011 & Total \\
\hline & n (\%) & n (\%) & n (\%) & n (\%) \\
\hline Total & $90(35,0)$ & $96(37,4)$ & $71(27,6)$ & $257(100,0)$ \\
\hline \multicolumn{5}{|l|}{ Sexo } \\
\hline Feminino & $39(43,3)$ & $50(52,1)$ & $31(43,7)$ & $120(46,7)$ \\
\hline Masculino & $51(56,7)$ & $46(47,9)$ & $40(56,3)$ & $137(53,3)$ \\
\hline Idade (média \pm DP) & $75,77 \pm 14,08$ & $76,54 \pm 12,97$ & $76,79 \pm 10,37$ & $76,34 \pm 12,67$ \\
\hline \multicolumn{5}{|l|}{ Dados clínicos na admissão (média \pm DP) } \\
\hline Pressão arterial sistólica & $161,01 \pm 30,23$ & $153,42 \pm 26,47$ & $156,42 \pm 30,03$ & $156,91 \pm 28,90$ \\
\hline Pressão arterial diastólica & $86,23 \pm 13,89$ & $85,97 \pm 15,30$ & $84,70 \pm 15,98$ & $85,71 \pm 14,97$ \\
\hline Frequência cardíaca & $76,53 \pm 15,37$ & $76,59 \pm 16,36$ & $75,77 \pm 15,30$ & $76,34 \pm 15,67$ \\
\hline Hemoglucoteste & $131,08 \pm 37,70$ & $136,15 \pm 49,34$ & $127,56 \pm 39,51$ & $132,02 \pm 48,84$ \\
\hline \multicolumn{5}{|l|}{ Comorbidades na admissão } \\
\hline Diabetes & $19(21,1)$ & $22(22,9)$ & $15(21,1)$ & $56(21,8)$ \\
\hline Fibrilação atrial & $22(24,4)$ & $21(21,9)$ & $14(19,7)$ & $57(22,2)$ \\
\hline Hipertensão arterial & $63(70,0)$ & $67(69,8)$ & $45(64,3)$ & $175(68,4)$ \\
\hline Doença arterial coronariana & $11(12,2)$ & $10(10,4)$ & $7(9,9)$ & $22(10,9)$ \\
\hline AVC/Ataque isquêmico transitório prévio & $28(31,1)$ & $26(27,1)$ & $14(19,7)$ & $68(26,5)$ \\
\hline \multicolumn{5}{|l|}{ Tipo de alta } \\
\hline Alta & $69(76,7)$ & $78(81,3)$ & $56(78,9)$ & $203(79,0)$ \\
\hline Óbito & $6(6,7)$ & $5(5,2)$ & $2(2,8)$ & $13(5,1)$ \\
\hline
\end{tabular}

AVC: acidente vascular cerebral; DP: desvio-padrão.

foi de $11,5 \%$. Não houve diferença estatisticamente significativa entre o percentual de homens $(7,3)$ e mulheres $(6,7)$ trombolisados.

A Tabela 1 apresenta o número de barreiras identificadas desde a entrada do paciente com hipótese diagnóstica de AVCi, independentemente de ainda ser, ou não, candidato à trombólise no momento de identificação da barreira. Do total de barreiras identificadas, somente 22 foram classificadas como barreiras ligadas direta- 
mente aos médicos. Dentre as barreiras externas, 31 foram classificadas como barreiras ligadas ao paciente. É importante notar que essas barreiras não são excludentes.

\section{Discussão}

O presente estudo examinou a ocorrência de barreiras à trombólise de casos que chegaram à emergência de um hospital privado com suspeita de AVCi. O hospital apresenta condições muito favoráveis à adoção da diretriz, no entanto observou-se que dos 20 pacientes que chegaram ao final do fluxo de cuidado como candidatos à trombólise, 9 (45\%) não foram trombolisados por existência de barreiras. A comparação desse percentual com outros estudos não pôde ser feita dadas as diferenças metodológicas.

Do total de pacientes com diagnóstico confirmado de AVCi (156), 11,5\% (18) foram trombolisados. Estimativas norte-americanas de 200921 indicam que 3,4\% a 5,2\% dos pacientes com AVCi receberam trombólise, aproximadamente o dobro da taxa de tratamento de 2005 e metade da observada no presente estudo. Esses resultados sugerem haver um melhor manejo do AVCi no serviço estudado, apesar do alto percentual de barreiras identificadas no final do fluxo de cuidado. Certamente, pensando-se em outros cenários brasileiros, a situação seria diferente. O percentual de não trombólise pela presença de barreiras seria bem maior e, provavelmente, o percentual de trombolisados entre aqueles com diagnóstico de AVCi seria bem menor.

Segundo a metanálise de Reeves et al. 22 com 18 estudos, mulheres possuem chance $30 \%$ menor de receberem trombólise em relação a homens, o que não foi verificado no presente estudo. A população brasileira apresenta características sociais e demográficas diferentes dos pacientes investigados, além de ser atendida por equipes com diferentes níveis de treinamento. Assim, o alerta para a tendência de se trombolisar menos mulheres deve ser considerado e avaliado, tendo em vista esse achado ser frequente em outras realidades.

A etnia do paciente também tem sido relacionada com diferenças na chance de trombólise. Segundo o estudo de Coutinho et al. ${ }^{19}$, realizado em Amsterdam, não brancos possuem uma chance de serem trombolisados 2,5 vezes menor que os brancos, sendo a demora em chegar ao hospital o principal fator associado. Segundos as leis do local do estudo, hospitais e médicos são obrigados a realizar o atendimento completo, independentemente do seguro de saúde, dessa forma os autores acreditam que as diferenças se devem mais provavelmente a um viés médico em relação à etnia. Estudos realizados nos Estados Unidos 23,24 também reportaram que negros possuem menor chance de trombólise que brancos. Não se investigou a relação da etnia com a trombólise neste estudo, contudo, em um país altamente miscigenado como o Brasil, esse aspecto deve ser enfatizado em treinamentos sobre o manejo do AVCi.

\section{Barreiras relacionadas diretamente ao médico}

Neste estudo, o não consentimento para a trombólise por parte do médico assistente (que não é funcionário do hospital) impediu o tratamento de dois pacientes em vinte elegíveis. Essa barreira já foi identificada em outros estudos 25,26 e sua ocorrência pode estar relacionada tanto à dificuldade clínica de comunicação do paciente (deficit de linguagem, redução do nível de consciência), quanto à incerteza do paciente ou médico assistente sobre os riscos e benefícios do tratamento, revelando mais uma vez a importância da falta de disseminação de conhecimento médico específico como barreira à trombólise.

Estudo realizado nos Estados Unidos 27 envolvendo médicos emergencistas demonstrou que uma parcela significativa dos profissionais possui receio de realizar a trombólise, sendo as principais razões citadas: receio de hemorragia intracerebral e sensação de falta de benefício da terapia trombolítica.

\section{Barreiras externas}

\section{- Fatores relacionados aos pacientes}

A barreira mais consistentemente reportada pela literatura está relacionada a fatores relacionados ao paciente, ou seja, a demora em chegar ao hospital após o início dos sintomas. Revela o baixo nível de conhecimento populacional a respeito do AVC, resultando em demora por busca de atendimento médico 28,29,30. Os principais motivos identificados pelos estudos são: paciente ou familiar não reconheceu sintomas 30,31,32,33,34,35 ou não buscou por ajuda 28,36,37 e paciente ou familiar não acionou o serviço pré-hospitalar 20,28,31,36,38. Entre esses, houve maior frequência de pacientes que moravam sozinhos ou não tinham um acompanhante no momento do início dos sintomas ${ }^{39}$, falta de senso de urgência pelo paciente ou familiar e recusa do paciente em ir para o hospital ${ }^{31}$. O estudo de Morris et al. ${ }^{40}$, realizado nos Estados Unidos, demonstrou que $44 \%$ dos pacientes chegavam ao hospital após 3 horas do início dos sintomas. Em nosso estudo, 39\% 
(80/206) dos pacientes com registro em prontuário do tempo do início dos sintomas chegaram à emergência com tempo superior ao recomendado pelo estudo NINDS, enquanto no estudo de Carvalho et al. 41, realizado em 19 hospitais públicos e privados de Salvador, Bahia, apenas $22 \%$ dos pacientes deram entrada no hospital com menos de 3 horas e 28,1\% com menos de 4,5 horas do início dos sintomas, o que evidencia a disparidade de reconhecimento dos sinais de alerta e busca pelo atendimento entre as populações de diferentes regiões brasileiras.

Além da falta de conhecimento do público leigo dos sintomas do AVC e da urgência em buscar tratamento, há dificuldade de acesso aos hospitais, falta de transporte e desinformação do médico assistente (médico da família). Pacientes transferidos de ambulância demoram menos a chegar ao hospital, enquanto os que entram em contato primeiro com o médico assistente chegam mais tardiamente. Em nosso estudo, o percentual de pessoas que chegaram dentro da janela terapêutica foi maior do que o dos estudos citados. Apesar de não se ter informação sobre o transporte utilizado pelo paciente, tempo do contato com o médico assistente, ou sobre a conduta inicial por ele preconizada, sabe-se que a população atendida pelo hospital do estudo tem boa escolaridade, bom nível de renda e reside em áreas com boa disponibilidade de transporte, especialmente táxis.

Ao serem avaliados os problemas de atendimento nas emergências do Brasil, o atendimento tanto em unidades privadas quanto públicas, as dificuldades citadas devem ser levadas em conta, e se deve pensar em tempos maiores de chegada ao hospital como mostra o estudo de Carvalho et al. ${ }^{41}$.

Segundo Quain et al. ${ }^{42}$, a parcela de pacientes que recebe rtPA poderia aumentar de 4,7\% para $21,4 \%$ com a implementação de protocolos e investimentos em educação, que estão relacionadas a uma redução da mediana de tempo do início dos sintomas de 150 para 90,5 minutos. Tal preocupação se aplica ao Brasil visto que, mesmo em uma população com bom nível de escolaridade como a estudada, presume-se que o reconhecimento dos sintomas/urgência foi baixo, dado o alto percentual de pacientes que chegaram após a janela terapêutica $(38,8 \%$ do total de pacientes atendidos com alguma referência ao tempo de início de sintomas). O poder público e os médicos deveriam criar, incentivar e apoiar iniciativas objetivando educar a população sobre os sinais de alerta do AVC com o objetivo de reduzir o tempo até o primeiro atendimento em uma unidade hospitalar, como já ocorre em vários países.

\section{- Barreiras externas: fatores relacionados à diretriz}

Não se relacionou nenhuma barreira identificada a problemas relacionados à diretriz porque o hospital adotou a diretriz, treinou e certificou seus médicos, o que é refeito regularmente.

\section{- Barreiras externas: fatores ambientais}

Através de cuidadosa busca no MEDLINE, não foram identificados artigos que analisassem a ausência de registro no prontuário do tempo do início dos sintomas, um dado crítico para a elegibilidade para trombólise. Classificou-se essa ausência como barreira ligada diretamente ao médico, por conta da falta de familiaridade com a diretriz, ou falta de motivação. Ocorreu em $20 \%$ dos prontuários de pacientes que chegaram à emergência com suspeita clínica de AVCi. Na revisão de Cabana et al. 16 sobre a adesão a diretrizes clínicas de várias áreas da medicina, o percentual mediano (valor mínimo e máximo) de respondentes, reportando a falta de familiaridade, foi de 56,5 (0-89) e falta de motivação, 42,0 (23-68). Os autores ressaltam que o simples conhecimento da diretriz não garante a familiaridade com as recomendações das diretrizes e a capacidade de aplicá-las corretamente. Citando teorias comportamentais, correlacionando com os resultados da revisão, Cabana et al. 16 ressaltam que em toda mudança há uma fase inicial quando é necessária uma força extra, profissional, pessoal e/ou social. Como foi dito em métodos, a emergência do hospital em estudo faz treinamentos periódicos sobre diretrizes clínicas, não existindo, porém, outros reforços como um sistema de lembretes, aconselhamento, ou reuniões de equipes. Há heterogeneidade de respostas às intervenções visando à diminuição do gap entre o conhecimento e a prática, em razão das diferenças organizacionais entre os hospitais. Ao se pensar nos hospitais públicos brasileiros, parece muito importante introduzir formas contínuas de incentivo à implementação da diretriz para trombólise em pacientes que chegam à emergência com suspeita de AVCi.

$\mathrm{Na}$ literatura, a mediana de retardo entre a admissão hospitalar e a primeira avaliação médica difere entre os centros hospitalares. Schroeder et al. 39 encontraram mediana de vinte minutos enquanto Feldmann et al. 30 encontraram mediana de quatro horas. No presente estudo, tal barreira não foi verificada, uma vez que o tempo médio de atendimento para qualquer paciente foi de oito minutos. Ao se extrapolar para a população brasileira, tal barreira certamente acontecerá, tendo em vista a provável discrepância no 
tempo de atendimento entre hospitais públicos e particulares de diferentes regiões do país, devido a barreiras ambientais: falta de tempo e/ou falta de recursos.

A falta de avaliação neurológica e o tempo de avaliação do neurologista superior a 270 minutos impediram a trombólise de dois pacientes. Esses casos também foram classificados como barreiras ambientais: falta de tempo e/ou falta de recursos.

O tempo de primeira imagem varia segundo o tempo de requisição do exame, transporte do paciente para o departamento de radiologia, análise do resultado pelo neurorradiologista. No estudo de Wester et al. 20, a mediana de tempo entre a chegada à emergência e a chegada à sala de tomografia foi de 2 horas e 54 minutos. No estudo de Lin et al. 43 , o tempo médio entre a chegada e a realização da tomografia foi de 58 minutos. Neste estudo, a mediana de tempo até a realização da primeira imagem foi de 53 minutos, tempo reduzido em relação ao encontrado em Fortaleza 41, com mediana de 3,4 horas entre a admissão hospitalar e o tempo de primeira imagem. Não se avaliou, todavia, se as etapas entre a requisição do exame e sua execução representaram um gasto desnecessário de tempo. Ressaltase que o tempo de primeira imagem apresentou uma correlação negativa com o NIHSS, mostrando que quanto maior a gravidade do caso, menor é o tempo até a realização do exame.

Grande parte da bibliografia sobre barreiras à adesão a diretrizes clínicas trabalha com questionários ou entrevistas com médicos, chefes e/ou gerentes de serviços, individualmente ou em grupos 17. Buscam captar a percepção dos profissionais. As revisões sobre o tema também incluem estudos sobre a opinião dos profissionais 16,17 . Poucos são os estudos que identificaram as barreiras através da análise do processo de cuidado, o que torna muito difícil a comparação dos dados expostos na Tabela 1. Por exemplo, um estudo com médicos generalistas holandeses investigou as barreiras percebidas à adesão a quatro diferentes diretrizes clínicas. Os escores diferiram segundo a diretriz, mas em média, $9 \%$ relataram haver falta de conhecimento ou familiaridade com as diretrizes e $16,9 \%$ mencionaram a falta de motivação. Quanto às barreiras externas, $23 \%$ citaram fatores relacionados aos pacientes e $18,8 \%$ constataram haver barreiras ambientais 18 . Esses percentuais são muito inferiores aos encontrados neste estudo no qual se identifica- ram pelo menos $158(61,48 \%)$ barreiras relacionadas somente a fatores ambientais.

As duas perspectivas de análise aqui adotadas têm limitações. Pode-se argumentar que uma vez que o paciente não seja mais candidato à trombólise, seja por barreira, perda da indicação ou identificação de uma contraindicação, o protocolo da trombólise não mais se aplica e o tempo entre as etapas de atendimento pode ser flexibilizado. Esse argumento se fortalece diante da constatação de que os tempos de primeira imagem e avaliação do neuro apresentaram correlação negativa com o NIHSS. Os dados da tabela estariam superestimando os percentuais de barreiras. Por outro lado, ao serem excluídos da contagem das barreiras, a cada passo do processo de cuidado, os pacientes que deixaram de ser candidatos à trombólise, poder-se-ia estar subestimando os percentuais das barreiras. Optou-se por apresentar e discutir os resultados nas duas perspectivas de análise.

O presente estudo tem limitações. A primeira delas é não se ter investigado o motivo da demora em chegar à emergência. Tal informação seria importante para orientar ações educativas para a população. Em segundo lugar, não foram investigados os motivos de demora em realização do primeiro exame de imagem, conhecimento que poderia informar mais sobre tal barreira. Também não se investigou a percepção dos médicos, assim a classificação das barreiras foi decidida pelos autores.

Em resumo, a identificação de barreiras é um item fundamental para possibilitar a melhoria dos processos assistenciais. Mesmo em condições favoráveis, observou-se que quase a metade dos pacientes que deveriam ter sido trombolisados não o foi por causa de barreiras. Nas emergências públicas do Brasil, a realidade seria muito mais adversa. Algumas recomendações podem ser extraídas do presente trabalho. A disponibilidade de serviços de imagem e de neurologistas nas emergências, que diminuam o tempo entre a chegada do paciente e o momento da trombólise, a necessidade de se criarem formas de incentivo ao conhecimento e familiaridade com a diretriz nos hospitais e para os médicos em geral, discutindo a sua eficácia; incentivo ao registro no prontuário do tempo do início dos sintomas; a criação de estratégias de educação de pacientes para reconhecerem os sintomas do AVC, sua gravidade e a necessidade de buscar imediatamente um serviço de emergência. 


\section{Resumen}

El accidente cerebrovascular isquémico (AVCi) es una causa importante de daño en tejidos y muerte. El uso correcto de trombolíticos se enfrenta a varios obstáculos. El objetivo fue analizar las barreras para el tratamiento trombolítico en pacientes que llegaran con síntomas de AVCi agudo al servicio de urgencias de un hospital privado de Río de Janeiro, Brasil. Se realizó un estudio de cohorte retrospectivo de los pacientes, entre los años 2009 y 2011. 257 pacientes fueron ingresados con sospecha de AVCi. De los pacientes con casos confirmados (156), 11,5\% (18) fueron trombolizados. De los 30 pacientes con un accidente cerebrovascular isquémico dentro de la ventana terapéutica y NIHSS correcto para la trombólisis, 20 no se trombolizaron, 9 por obstáculos. En nuestro estudio el porcentaje de trombólisis fue mayor que en los Estados Unidos, pero las barreras impidieron el tratamiento de un $45 \%$ de los pacientes con una indicación de trombólisis.

Accidente Cerebrovascular; Terapia Trombolítica; Calidad de la Atención de Salud

\section{Colaboradores}

L. H. O. Fonseca, M. L. G. Rosa, A. C. Silva, R. M. Maciel, A. Volschan e E. T. Mesquita participaram com contribuições substanciais à concepção e desenho do artigo, à análise e interpretação dos dados, à redação e à aprovação final da versão a ser publicada.

\section{Referências}

1. Manobianca G, Zoccolella S, Petruzzellis A, Miccoli A, Logroscino G. Low incidence of stroke in southern Italy: a population-based study. Stroke 2008; 39:2923-8.

2. Cabral NL, Gonçalves AR, Longo AL, Moro CH, Costa G, Amaral CH, et al. Incidence of stroke subtypes, prognosis and prevalence of risk factors in Joinville, Brazil: a 2 year community based study. J Neurol Neurosurg Psychiatry 2009; 80:755-61.

3. André C, Curioni CC, Cunha CB, Veras R. Progressive decline in stroke mortality in Brazil from 1980 to 1982,1990 to 1992 , and 2000 to 2002 . Stroke 2006; 37:2784-9.

4. Brott T, Bogousslavsky J. Treatment of acute ischemic stroke. N Engl J Med 2000; 343:710-22.

5. Sociedade Brasileira de Doenças Cerebrovasculares. Primeiro consenso brasileiro para trombólise no acidente vascular cerebral isquêmico agudo. Arq Neuropsiquiatr 2002; 60:675-80.

6. Adams Jr. HP, del Zoppo G, Alberts MJ, Bhatt DL, Brass L, Furlan A, et al. Atherosclerotic peripheral vascular disease working group: quality of care outcomes in research interdisciplinary working group. Circulation 2007; 115:e478-534.

7. Tissue plasminogen activator for acute ischemic stroke. The National Institute of Neurological Disorders and Stroke rt-PA Stroke Study Group. N Engl J Med 1995; 333:1581-7.
8. Conforto AB, Paulo RB, Patroclo CB, Pereira SL, Miyahara HS, Fonseca CB, et al. Stroke management in a university hospital in the largest South American city. Arq Neuropsiquiatr 2008; 66: 308-11.

9. Katzan IL, Hammer MD, Hixson ED, Furlan AJ, Abou-Chebl A, Nadzam DM, et al. Utilization of intravenous tissue plasminogen activator for acute ischemic stroke. Arch Neurol 2004; 61:346-50.

10. Hill MD, Buchan AM. Thrombolysis for acute ischemic stroke: results of the Canadian Alteplase for Stroke Effectiveness Study. CMAJ 2005; 172: 1307-12.

11. Massaro AR. Stroke in Brazil: a South America perspective. Int J Stroke 2006; 1:113-5.

12. Pontes-Neto OM, Silva GS, Feitosa MR, Figueiredo NL, Fiorot Jr. JA, Rocha TN, et al. Stroke awareness in Brazil: alarming results in a community-based study. Stroke 2008; 39:292-6.

13. Hacke W, Kaste M, Bluhmki E, Brozman M, Dávalos A, Guidetti D, et al. Thrombolysis with alteplase 3 to 4.5 hours after acute ischemic stroke. N Engl J Med 2008; 359:1317-29.

14. Audet AM, Greenfield S, Field M. Medical practice guidelines: current activities and future directions. Ann Intern Med 1990; 30:709-14.

15. Chassin MR. Practice guidelines: best hope for quality improvement in the 1990s. J Occup Med 1990; 32:1199-206. 
16. Cabana MD, Rand CS, Powe NR, Wu AW, Wilson $\mathrm{MH}$, Abboud PAC, et al. Why don't physicians follow clinical practice guidelines? A framework for improvement. JAMA 1999; 282:1458-65.

17. Cochrane LJ, Olson CA, Murray S, Dupuis M, Tooman T, Hayes S. Gaps between knowing and doing: understanding and assessing the barriers to optimal health care. J Contin Educ Health Prof 2007; 27:94-102.

18. Lugtenberg M, Burgers JS, Besters CF, Han D, Westert GP. Perceived barriers to guideline adherence: a survey among general practitioners. BMC Fam Pract 2011; 12:98.

19. Coutinho JM, Klaver EC, Roos YB, Stam J, Nederkoorn PJ. Ethnicity and thrombolysis in ischemic stroke: a hospital based study in Amsterdam. BMC Neurol 2011; 11:81.

20. Wester P, Rådberg J, Lundgren B, Peltonen M. Factors associated with delayed admission to hospital and in-hospital delays in acute stroke and TIA: a prospective, multicenter study. Seek-MedicalAttention-in-Time Study Group. Stroke 1999; 30: 40-8.

21. Adeoye O, Hornung R, Khatri P, Kleindorfer D. Recombinant tissue-type plasminogen activator use for ischemic stroke in the United States: a doubling of treatment rates over the course of 5 years. Stroke 2011; 42:1952-5.

22. Reeves M, Bhatt A, Jajou P, Brown M, Lisabeth L. Sex differences in the se of intravenous rtPA thrombolysis treatment for acute ischemic stroke: a meta-analysis. Stroke 2009; 40:1743-9.

23. Johnston SC, Fung LH, Gillum LA, Smith WS, Brass $\mathrm{LM}$, Lichtman JH, et al. Utilization of intravenous tissue-type plasminogen activator for ischemic stroke at academic medical centers: the influence of ethnicity. Stroke 2001; 32:1061-8.

24. Schwamm LH, Reeves MJ, Pan W, Smith EE, Frankel MR, Olson D, et al. Race/ethnicity, quality of care, and outcomes in ischemic stroke. Circulation 2010; 121:1492-501.

25. Grond M, Stenzel C, Schmülling S, Rudolf J, Neveling $M$, Lechleuthner A, et al. Early intravenous thrombolysis for acute ischemic stroke in a community-based approach. Stroke 1998; 29:1544-9.

26. André C, Moraes-Neto JB, Novis SA. Experience with t-PA treatment in a large South American city. J Stroke Cerebrovasc Dis 1998; 7:255-8.

27. Brown DL, Barsan WG, Lisabeth LD, Gallery ME Morgenstern LB. Survey of emergency physicians about recombinant tissue plasminogen activator for acuteischemic stroke. Ann Emerg Med 2005; 46:56-60.

28. Kothari R, Jauch E, Broderick J, Brott T, Sauerbeck L, Khoury J, et al. Acute stroke: delays to presentation and emergency department evaluation. Ann Emerg Med 1999; 33:3-8.

29. Jørgensen HS, Nakayama H, Reith J, Raaschou HO, Olsen TS. Factors delaying hospital admission in acute stroke: the Copenhagen Stroke Study. Neurology 1996; 47:383-7.
30. Feldmann E, Gordon N, Brooks JM, Brass LM, Fayad PB, Sawaya KL, et al. Factors associated with early presentation of acute stroke. Stroke 1993; 24:1805-10

31. Ferro JM, Melo TP, Oliveira V, Crespo M, Canhão P, Pinto AN. An analysis of the admission delay of acute strokes. Cerebrovasc Dis 1994; 4:72-5.

32. Pistollato G, Ermani M. Time of hospital presentation after stroke:a multicenter study in north-east Italy. Italian SINV (Società Interdisciplinare Neurovascolare) Study group. Ital J Neurol Sci 1996; 17:401-7.

33. Alberts MJ, Bertels C, Dawson DV. An analysis of time of presentation after stroke. JAMA 1990; 263:65-8.

34. Biller J, Patrick JT, Shepard A, Adams HP. Delay time between onset of ischemic stroke and hospital arrival. J Stroke Cerebrovasc Dis 1993; 3:228-30.

35. Duncan PW, Harrison DJ, Lai SM, Cook SF, Rymer MM. Factors affecting elapsed time between acute stroke and presentation. Stroke 2000; 31:308.

36. Siu YC, Wong TW, Lau CC. Candidates for thrombolytic treatment in acute ischaemic stroke: where are our patients in Hong Kong? J Accid Emerg Med 1999; 16:412-7.

37. Ravindrane A, Croft-Baker J, Jarrett D, Severs MP. Causes of delay in hospital assessment after stroke. Age Ageing 2000; 29 Suppl 2:57.

38. Lacy CR, Suh DC, Bueno M, Kostis JB. Delay in presentation and evaluation for acute stroke: Stroke Time Registry for Outcomes Knowledge and Epidemiology (S.T.R.O.K.E.). Stroke 2001; 32:63-9.

39. Schroeder EB, Rosamond WD, Morris DL, Evenson KR, Hinn AR. Determinants of use of emergency medical services in a population with stroke symptoms: the Second Delay in Accessing Stroke Healthcare (DASH II) Study. Stroke 2000; 31: 2591-6.

40. Morris DL, Rosamond W, Madden K, Schultz C, Hamilton S. Prehospital and emergency department delays after acute stroke: the Genentech Stroke Presentation Survey. Stroke 2000; 31: 2585-90.

41. Carvalho, Alves MB, Viana GA, Machado CB, Santos BF, Kanamura AH, et al. Stroke epidemiology, patterns of management, and outcomes in Fortaleza, Brazil: a hospital-based multicenter prospective study. Stroke 2011; 42:3341-6.

42. Quain DA, Parsons MW, Loudfoot AR, Spratt NJ, Evans MK, Russell ML, et al. Improving access to acute stroke therapies:a controlled trial of organised pre-hospital and emergency care. Med J Aust 2008; 189:429-33.

43. Lin CS, Tsai J, Woo P, Chang H. Prehospital delay and emergency department management of ischemic stroke patients in Taiwan, R.O.C. Prehosp Emerg Care 1999; 3:194-200.

Recebido em 04/Dez/2012

Versão final reapresentada em 06/Jun/2013 Aprovado em 03/Jul/2013 